\title{
Representations underlying skill in the discrete sequence production task: effect of hand used and hand position
}

\author{
Elian de Kleine $\cdot$ Willem B. Verwey
}

Received: 29 October 2007 / Accepted: 3 October 2008 / Published online: 5 November 2008

(C) The Author(s) 2008. This article is published with open access at Springerlink.com

\begin{abstract}
Various studies suggest that movement sequences are initially learned predominantly in effectorindependent spatial coordinates and only after extended practice in effector-dependent coordinates. The present study examined this notion for the discrete sequence production (DSP) task by manipulating the hand used and the position of the hand relative to the body. During sequence learning in Experiment 1, in which sequences were executed by reacting to key-specific cues, hand position appeared important for execution with the practiced but not with the unpracticed hand. In Experiment 2 entire sequences were executed by reacting to one cue. This produced similar results as in Experiment 1. These experiments support the notion that robustness of sequencing skill is based on several codes, one being a representation that is both effector and position dependent.
\end{abstract}

\section{Introduction}

Most actions we perform in everyday life exist of series of simple movements. For example, we lace our shoes in one fluent movement while it actually consists of a series of several more simple movements. This illustrates that we can sequence simple movements in a specific order to attain fluent execution of more complex movement patterns. Recent research suggests that multiple processors may be active during the execution of a movement sequence and

E. de Kleine $(\bowtie) \cdot$ W. B. Verwey

Cognitive Psychology and Ergonomics,

Faculty of Behavioral Sciences, University of Twente,

Postbus 217, 7500 AE Enschede, The Netherlands

e-mail: e.dekleine@utwente.nl that each processor involves another type of representation that, in addition, develops after varying amounts of practice (Hardy et al. 1996; Park and Shea 2005; Ungerleider et al. 2002; Verwey 2003). For example, skilled movement sequences have been shown to involve spatial and nonspatial information (Bapi et al. 2000; Koch and Hoffmann 2000a; Mayr 1996) as well as effector-dependent and effector-independent components (Hikosaka et al. 1999; Verwey 2003). It is generally accepted that sequence learning develops through various learning phases, from an initial attentive phase to an automatic phase, in which no attention is needed to perform the movement. This has been described also as a transition from the declarative phase to the procedural phase (Fitts 1964; Anderson 1982). For example, without practice full attention is needed to lace a shoe, but after practice the hands seem to know how to execute the task. Yet, evidence for the different representations and their role at various stages of skill remains scattered and people may well be flexible at switching from one to another representation (Verwey 2003).

Hikosaka et al. (1999) proposed a model in which sequence learning is acquired independently by two parallel systems; one using the spatial system and one using the motor system. The spatial system is assumed to be predominantly active at the early stages of sequence learning and involves knowledge of individual sequence elements in codes that are not effector-dependent. The motor system is assumed to be primarily active at the later stages of sequence learning and movement skill is assumed to involve effector-dependent sequence knowledge. Both systems learn the sequence independently and are assumed to be simultaneously active. However, Hikosaka et al. (1999) propose that the level of system activation varies across practice and either sequence mechanism may have a more important contribution, depending on the behavioral 
context. An additional feature of their model is that during execution of a movement sequence the motor system can learn from the spatial system and visa versa.

In extension to the Hikosaka et al. (1999) model, Bapi et al. (2000) distinguished an effector-dependent and an effector-independent sequence representation. They suggest that the effector-dependent representation is acquired relatively slowly by the motor system and that the effectorindependent representation is in visual/spatial coordinates and acquired relatively fast. In a later study, Bapi et al. (2006) provided evidence that different cortical and subcortical networks are engaged at various stages of learning which supported the notion of different sequence representations. The Hikosaka et al. (1999) model suggests that, in what they call the pre-learning stage, each stimulus leads to a movement without any effect of preceding or subsequent stimuli and therefore each movement relies on an individual sensorimotor transformation. However, during repeated execution of movement patterns representations develop that code the order of the individual movements. This would occur for the spatial and for the motor system, resulting in a spatial sequence and a motor sequence. The Hikosaka et al. (1999) model assumes that the spatial sequence is acquired relatively quickly and the motor sequence is acquired more slowly.

In order to differentiate the reliance on different types of sequence representations, Verwey (2003) analyzed response time distributions of a sequence learning task. His analysis of response time distributions was in line with the notion that during practice various processing modes had developed and that participants can switch from one to another processing mode as a function of whether the forthcoming sequence is expected to be familiar. On basis of the response time distributions, Verwey (2003) distinguished (at least) three processing modes, a fast sequence mode possibly involving sequence learning at the motor level, a moderately fast mode perhaps involving sequence learning at a spatial level, and a slow mode that may well involve reacting to individual key-specific cues. The fast and the moderately fast modes correspond to the two stages of the Hikosaka et al. (1999) model and the slow processing mode corresponds to the pre-learning stage mentioned by Hikosaka et al. (1999). In addition, some processors may simultaneously race to determine which will trigger the next response, but support for parallel racing was limited (Verwey 2003).

To make the picture more complicated, a distinction has been made between spatial representations with an egocentric (i.e., a body-based reference frame) and allocentric (i.e., a world-based reference frame) representations. Egocentric reference frames may be eye-, hand- or body-centered (Colby and Goldberg 1999). Execution of spatial tasks is probably based on a mixture of representations with different reference frames (Adam et al. 2003; Heuer and Sangals 1998; Liu et al. 2007; Deroost et al. 2006). It is likely that depending on the task at hand, there are dominant processors and representations, and that with practice the contributions of these processors to sequence execution change.

In conclusion, there is a series of findings now indicating that executing movement sequences involves at least three mechanisms that may contribute simultaneously at advanced skill levels. First, when sequence execution involves responding to key-specific cues and there is no practice, control is entirely external and involves reacting to individual key-specific cues. Second, with limited practice, sequence control is based on effector-independent spatial coordinates, which may involve various representations with different reference frames. Third, with extensive practice, effector-dependent knowledge develops at the motor level. At this stage sequence execution may be based on one processor, but also on a mixture of independent spatial and motor processors that are alternated or racing to trigger responses.

In the present study we wanted to determine whether these various components are susceptible to the spatial location at which the sequence is carried out. The contribution of effector-dependent representations can be assessed by performance with the unpracticed effector. Previous research by Verwey and Wright (2004) provided support for the development of an effector-dependent component and for an effector-independent component during practice in the discrete sequence production (DSP) task. They showed that practiced sequences were performed faster with the practiced hand configuration than with an unpracticed hand configuration, suggesting an effector-dependent component, and that the practiced sequences were performed faster than new sequences with the unpracticed hand configuration, suggesting an effector-independent component. In a later study, Verwey and Clegg (2005) showed that the effector-dependent component also developed during the serial reaction-time task. They suggest that this effector-dependent component developed as a result of the extended practice they had used in their experiment, which is unusual in the serial reaction-time task. However, these studies did not investigate the contributions of spatial representations to effector-dependent and effector-independent sequence learning. The contribution of the spatial representation can be examined by transferring an acquired sequencing skill from one spatial configuration to another. A study by Grafton et al. (1998) showed that participants, executing the serial reaction-time task, are capable of transferring their skill from a normal to a large keyboard. This suggests that sequence knowledge can be represented on a relatively abstract level, independent of muscles used to respond and independent of the spatial representation. In 
contrast, a study by Rieger (2004) investigated the spatial representation during skilled typing with crossed hands and showed that typing skill involves a spatial representation. The models of Hikosaka et al. (1999) and Verwey (2003) suggest that effector-independent sequence learning is influenced by spatial coordinates because it is not related to specific body parts, while effector-dependent sequence learning is not influenced by spatial coordinates because it is related to specific body parts. However, to our knowledge this has not yet been investigated.

In the present study we used the DSP task which is thought to stimulate the development of an effector-dependent component because a discrete sequence of limited length is practiced thoroughly (Verwey and Wright 2004). In a typical DSP task two discrete sequences are practiced by responding to fixed series of three to six key-specific stimuli. All but the first stimuli are presented immediately after the response to the previous stimulus. In the present study each participant practiced two 7-key DSP sequences with their left hand. In order to test for effector-dependent and effector-independent sequence learning, the hand used to execute the sequence was varied during test phase. In order to examine the role of spatial representations on sequence execution the position of the keyboard on which the participants responded was also varied during the test phase. During the practice phase the keyboard was either placed $90^{\circ}$ to the left side of the body or $90^{\circ}$ to the right side of the body while the test phase involved both positions. So, during the practice phase participants practiced two sequences with their left hand, with the keyboard either at the left or the right side of their body. The test phase involved a 2 (Hand: practiced/left vs. unpracticed/ right) $\times 2$ (Keyboard position: familiar vs. unfamiliar $) \times 2$ (Sequence: familiar vs. random) between blocks design to examine transfer to the unpracticed hand and the unpracticed keyboard position. The independent variable Sequence was only used in Experiment 1.

In addition, the DSP is highly suitable to study sequence segmentation (Rhodes et al. 2004). Previous studies have shown that longer sequences consist of independent segments, which are thought to represent motor chunks (Verwey 2001; Verwey et al. 2002). In line with Allport (1980), Schmidt (1988) and Shaffer (1991), Verwey (2001) proposed that a cognitive and a motor component may underlie DSP. The cognitive component is thought to select a sequence (or chunk), based on a symbolic representation, and this sequence (or chunk) is read and executed by the motor component. The cognitive component additionally plans and organizes the goal structure of movements (Shaffer 1991). Based on this model it could be suggested that chunk execution is more susceptible to the spatial location at which the sequence is carried out than chunk transition, as chunk execution probably relies on a motoric representation becomes effector-dependent with practice. Therefore additional analyses were performed to investigate the contribution of a spatial representation to the different phases (chunk execution and chunk transition) of sequence execution.

In short, the purpose of the present experiments was to determine the spatial nature of effector-dependent and effector-independent representations at more advanced levels of sequence learning, by varying the hand and the position of the hand, relative to the body. Experiment 2 was conducted to replicate the results of Experiment 1 and to ascertain that the effects found in Experiment 1 had not been caused by different stimulus-response mappings in the two keyboard location conditions. That is, in Experiment 1 every key press was indicated by a cue and changing keyboard position implied a change in stimulus-response mapping too, the possible role of which was excluded in Experiment 2.

\section{Experiment 1}

\section{Method}

\section{Participants}

Thirty-two students (12 men, 20 women) from the University of Twente served as participants in this experiment. All were right-handed and between 18 and 27 years old. They received course credits for their participation.

\section{Apparatus}

Stimulus presentation and response registration were controlled by E-Prime 1.1 on a $2.8 \mathrm{GHz}$ Pentium 4 PC running under Windows XP. Participants were seated in a dimly lit room in front of a computer screen. A chinrest was used to ensure a constant viewing distance of $45 \mathrm{~cm}$ and a fixed head position. The keyboard was positioned in a holder either on a table $90^{\circ}$ to the left side of the body, or on a table $90^{\circ}$ to the right side of the body, depending on the condition (see Fig. 1).

\section{Task}

The display showed four horizontally aligned squares that functioned as placeholders for the stimuli. The squares were $2.8 \mathrm{~cm}$ long and wide and there was $0.4 \mathrm{~cm}$ between the squares. The four squares were drawn in silver and appeared in the center of the screen on a black background. At the start of a sequence the squares were filled with the background color (black). After a random time interval between 500 and $1,000 \mathrm{~ms}$ one square was filled with blue 

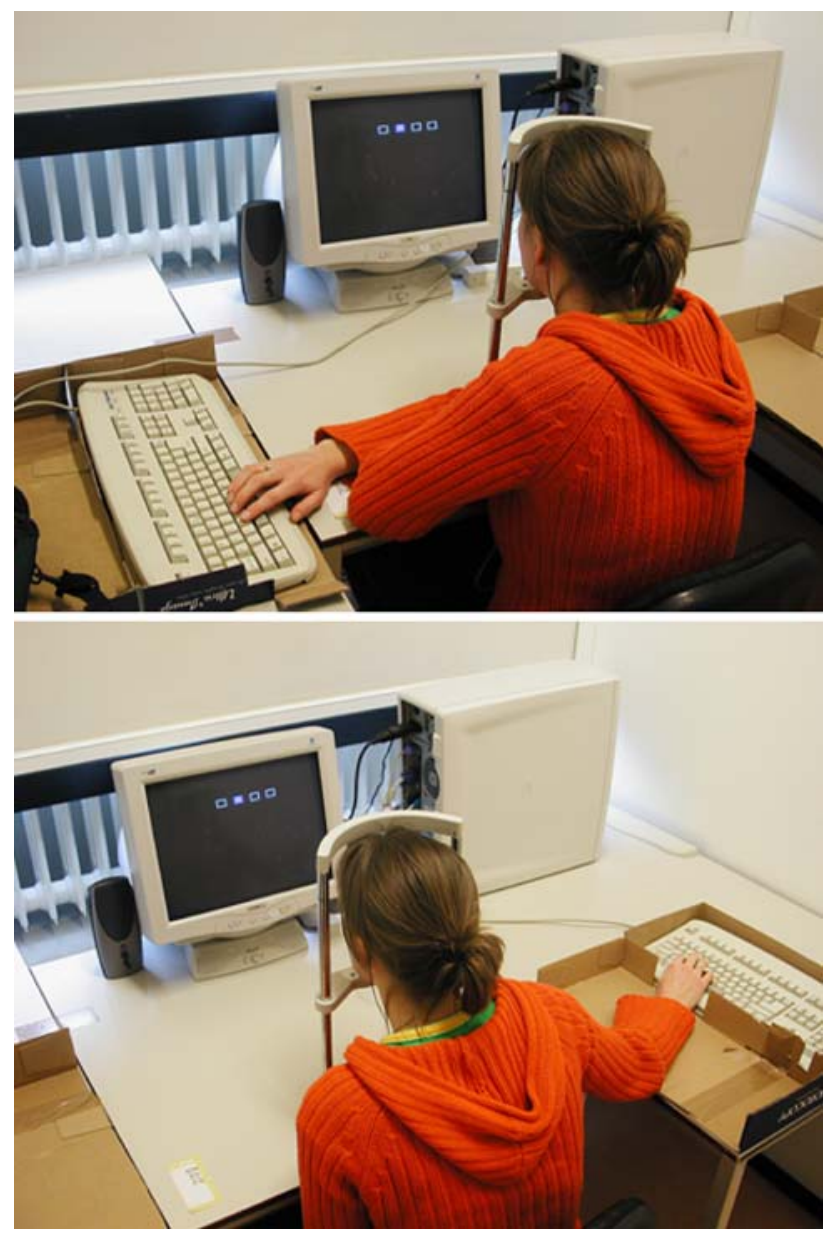

Fig. 1 Illustrations of a participant who executed the sequence with her right hand and the keyboard on the left side of the body (top) and with the keyboard on the right side of the body (bottom)

or purple, to which the participant reacted by pressing the corresponding key (to facilitate sequence learning two colors were used to differentiate between the two sequences). Immediately after a key press another square was filled, and so on. If a participant pressed a wrong key, an error message was given and the same square was filled again until the correct response was given. A premature first response was followed by feedback indicating that the response was too early, and the random foreperiod started again. One sequence involved seven successively filled squares and responses. After execution of a sequence the next sequence started, again with the four squares filled with black for a random time interval between 500 and $1,000 \mathrm{~ms}$.

Experimental conditions and counterbalancing variables are listed in Table 1. In this experiment four sequences were used, vnbnvbc, nvcvncb, bcncbnv, cbvbcvn, which are all characterized by the structure 1232134 . Half of the participants (16 participants) were assigned to Group 1 and executed sequences vnbnvbc and nvcvncb, the other half of the participants were assigned to Group 2 and executed sequences bencbnv and cbvbcvn. In the test phase, participants executed random sequences in addition to the practiced sequences. Executing one sequence was denoted a trial. The random sequences consisted of a random order of seven filled squares, which changed from trial to trial and were made up of the same four stimuli as the practiced sequences. In the random sequences a stimulus was never immediately repeated.

\section{Procedure}

During the practice phase the stimuli were arranged in four blocks of 80 sequences (40 repetitions of each sequence), yielding a total of 160 repetitions of each sequence during practice. Halfway through each block there was a break of $20 \mathrm{~s}$, during which the participant could relax. During this break and at the end of each block the participants received feedback about their mean response time and the number of errors since the previous feedback. Every practice block and every two test blocks were followed by a break that lasted approximately as long as a practice block (10 min).

Table 1 Experimental conditions and counterbalancing variables in Experiment 1 and 2

\begin{tabular}{lllll}
\hline Participant & $\begin{array}{l}\text { Experiment } 1 \text { and } 2 \\
\text { Practice side }\end{array}$ & $\begin{array}{l}\text { Experiment } 1 \text { and } 2 \\
\text { Sequence }\end{array}$ & $\begin{array}{l}\text { Experiment } 1 \text { and } 2 \\
\text { Test phase conditions }\end{array}$ & $\begin{array}{l}\text { Experiment } 1 \\
\text { Test phase order }\end{array}$ \\
\hline $1-4$ & Left & vnbnvbc, nvcvncb & Lh-Rs, Lh-Ls, Rh-Rs, Rh-Ls & Practice-Random \\
$5-8$ & Left & vnbnvbc, nvcvncb & Lh-Rs, Lh-Ls, Rh-Rs, Rh-Ls & Random-practice \\
$9-12$ & Right & vnbnvbc, nvcvncb & Lh-Rs, Lh-Ls, Rh-Rs, Rh-Ls & Practice-random \\
$13-16$ & Right & vnbnvbc, nvcvncb & Lh-Rs, Lh-Ls, Rh-Rs, Rh-Ls & Random-practice \\
$17-20$ & Left & bcncbnv, cbvbcvn & Lh-Rs, Lh-Ls, Rh-Rs, Rh-Ls & Practice-random \\
$21-24$ & Left & bcncbnv, cbvbcvn & Lh-Rs, Lh-Ls, Rh-Rs, Rh-Ls & Random-practice \\
$25-28$ & Right & bcncbnv, cbvbcvn & Lh-Rs, Lh-Ls, Rh-Rs, Rh-Ls & Practice-random \\
$29-32$ & Right & bcncbnv, cbvbcvn & Lh-Rs, Lh-Ls, Rh-Rs, Rh-Ls & Random-practice \\
\hline
\end{tabular}

$L h$-Rs left hand-right side, $L h$ - $L s$ left hand-left side, $R h$ - $R s$ right hand-right side and $R h-L s$ right hand-left side. The order of the test phase conditions in Experiment 1 was counterbalanced across participants 
Half of the participants in each sequence-group (eight participants) practiced with their left hand on the keyboard on the left side of their body and the other half practiced with their left hand on the keyboard on the right side. In the test phase sequence blocks (practiced-random or random-practice) were counterbalanced across participants.

During practice (and in half the test blocks), participant placed their left little finger on the C-key, their left ring finger on the V-key, their left middle finger on the B-key, and their left index finger on the N-key of a normal computer keyboard. In the remaining blocks of the test phase participants used their right hand, in which case the index finger was on the C-key, the middle finger on the V-key, the ring finger on the B-key and the little finger on the N-key. The four response keys had the same alignment on the keyboard as the four stimulus squares on the display. The instruction was to react as accurately and fast as possible.

\section{Data analysis}

The first two trials of every block, the first two trials after every break, and trials in which one or more errors had been made, were excluded from analyses. Sequences in which the total response time lasted longer than the mean sequence execution time across participants and within blocks plus three standard deviations were also eliminated from the analysis. This last procedure removed $1.1 \%$ of the trials. The Greenhouse-Geisser correction was used with corrected values of the degrees of freedom, when the sphericity assumption of the $F$-test was violated. Response time (RT) was defined as the time between the onsets of two consecutive key presses within a sequence (stimulus onset co-occurred with depression of the previous key). The time between onset of the first stimulus and depression of the first key was not included in the analyses, as this stimulus is preceded by an intertrial interval which makes it qualitatively different from the other responses. Mean RTs and arcsine transformed error rates across keys within a sequence were evaluated statistically by analysis of variance (ANOVA) with repeated measures, with in the practice phase Block (4) and Key (6) as within subject factors, and in the test phase Sequence (practiced vs. random sequence), Hand (practiced left hand vs. unpracticed right hand), Position (practiced vs. unpracticed) and Key (6) as within subject factors.

Results

\section{Practice phase}

Figure 2 shows that participants became faster with practice, $F(3,93)=290.0, P<0.001$, that some keys were executed faster than others, $F(5,155)=6.6, P<0.001$. Mean

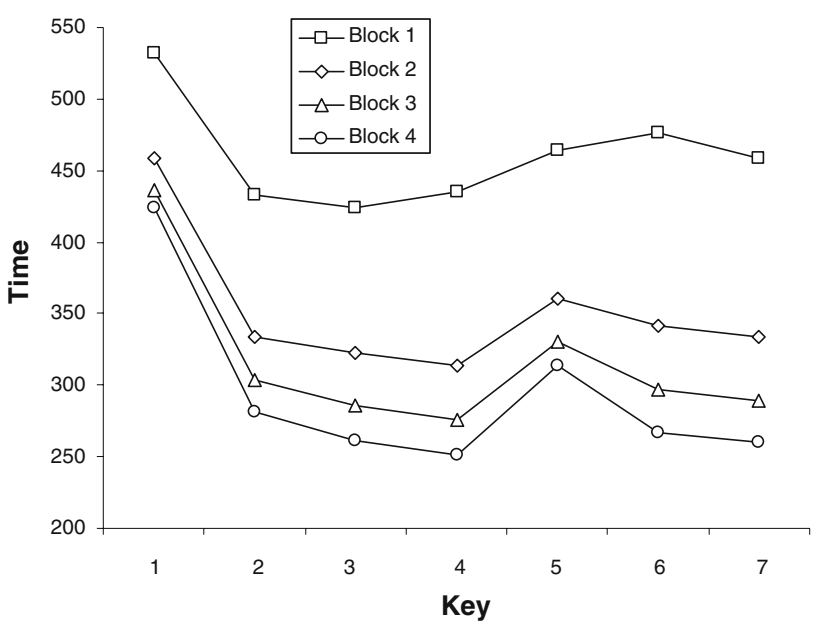

Fig. 2 Mean initiation time and mean RTs (in millisecond) across the two sequences in the four practice blocks of Experiment 1 as a function of key position within the sequence

error rate per key press amounted to $2 \%$ for the practice phase and some keys produced more errors than others, $F(5,155)=6.7, P<0.001$. The interaction between Block and Key on RT signified that gradually two segments developed, $F(15,465)=7.1, P<0.001$. This segmentation was confirmed by planned comparisons that indicated that in block 4 Key 5 was slower than Keys 2,3,4,6 and 7, $F s(1,31)>10.2, p s<0.005$. Furthermore, an additional ANOVA also including Group as independent variable showed that there was no significant interaction between Block, Key and Group $[F(15,450)=1.3, P>0.2]$, suggesting that the sequences had been identically segmented across participant groups, despite the two groups practicing different sequences. Summarizing, participants learned the sequences and with practice two segments developed.

\section{Test phase}

Practiced sequences were executed faster than random sequences, $F(1,31)=219.3, P<0.001$, and fewer errors were made in practiced sequences $(2 \%)$ than in random sequences $(3 \%), F(1,31)=8.6, P<0.005$. Sequences were executed faster with the practiced (left) hand than with the unpracticed (right) hand, $F(1,31)=7.5, P<0.01$, and the practiced hand $(2 \%)$ made less errors than the unpracticed hand $(3 \%), F(1,31)=8.0, P<0.01$. The differences in RT between the practiced and the unpracticed hand were bigger during the execution of practiced sequences than during the execution of random sequences, as was shown by the twoway interaction between Sequence and Hand, $F(1,31)=62.4$, $P<0.001$. This demonstrated effector-dependent sequence learning.

Sequence execution in the DSP task involves chunking (grouping of information), which results in segments of 


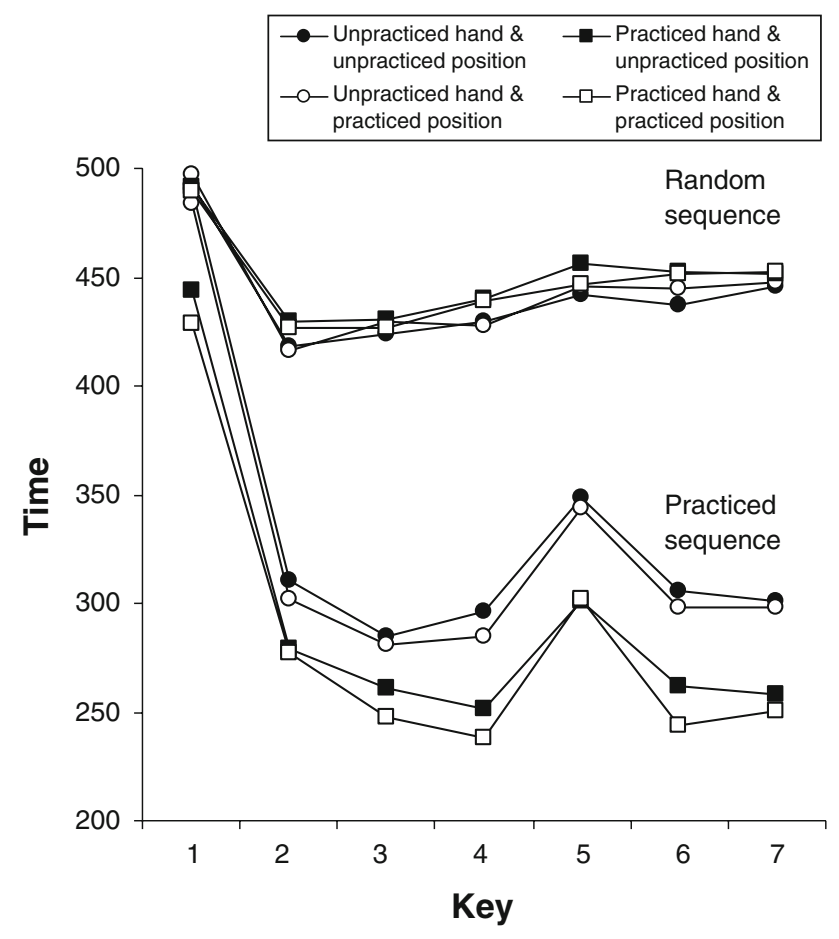

Fig. 3 Mean initiation time and mean RTs (in millisecond) in the test phase of Experiment 1 as a function of key position within the sequence, the condition within the test phase, the hand used and the position of the hand used

keys within sequences. Figure 3 shows that some keys were executed faster than others, $F(5,155)=12.6, P<0.001$, and that in the practiced sequence the RT differences between the keys were bigger than in the random sequence, $F(5,155)=9.8, P<0.001$. Given the obvious segmentation of the practiced sequences, the execution of the practiced sequences during the test phase was analyzed with a 2 (Hand; practiced left hand vs. unpracticed right hand) $\times 2$ (Position; practiced vs. unpracticed position) $\times 2$ (Phase; $\mathrm{T} 5 \rightarrow$ transition, $\mathrm{T} 2, \mathrm{~T} 3, \mathrm{~T} 4, \mathrm{~T} 6, \mathrm{~T} 7, \rightarrow$ execution) repeated measures ANOVA on mean RT. Results showed that the transition phase was significantly slower than the execution phase, $F(1,31)=24.7, P<0.001$. Planned comparisons were performed to investigate the relationship between the two phases and the spatial position. Most importantly, these planned comparisons showed that for the practiced sequences executed with the practiced hand there was a significant difference between the practiced and the unpracticed keyboard position for the execution phase, $F(1,31)=5.6, P<0.05$, and not for the transition phase, $F(1,31)=0.1, P=0.98$. Furthermore, the keyboard position did not influence the execution of practiced sequences with the unpracticed hand in either phase, $F(1,31)>0.2, P>0.3$. Apparently, the unfamiliar position of the practiced hand slowed the execution and not the transition phase of the practiced sequence.
Taken together, the practice phase showed that sequences were learned, became more clearly segmented with practice and that the sequences were identically segmented across participants and sequences. The test phase showed effector-dependent sequence learning. Finally, the position of the practiced hand affected the execution of chunks during effector-dependent sequence learning of the practiced sequences, and not transition.

\section{Discussion}

The aim of the present study is to examine whether the contribution of effector-dependent and effector-independent representations is influenced by the spatial position of the effector. Previous research suggested that sequences are initially learned in terms of effector-independent spatial coordinates, but later in practice sequences become increasingly effector-dependent (Bapi et al. 2000; Hikosaka et al. 1999; Verwey and Wright 2004). Our results confirmed that during practice effector-dependent sequence execution developed in that the unpracticed (right) hand was slower than the practiced (left) hand. No effect of spatial position across keys was found on effector-dependent and effector-independent sequence learning.

It turned out that the sequences used in this study were segmented at Key 5. The identical segmentation across sequences and across participant groups could be caused by the regularity imposed by the reversal in keys 2 until 4 (before the beginning of the second segment), as was also found in Koch and Hoffmann (2000b). Other causes are also possible; see Verwey and Eikelboom (2003). Anyway, because of this identical segmentation across participant groups and sequences two phases of sequence execution could be identified; e.g., chunks transition (T5) and chunk execution (T2, T3, T4, T6 and T7). The results showed that the execution phase during hand dependent sequence execution was influenced by the position of the hand and not the transition phase. This suggests that the elements within a chunk are stored in terms of spatial coordinates, whereas the first element of a chunk is not. Thus, although no effect of the spatial position across keys was found, analyzing the execution and transition phase of chunk independently it showed that the position of the hand influenced the execution of the chunk and not the transition.

A point of consideration is that the comparison of practice vs. random sequences was confounded with a variation in stimulus/response frequencies. The practiced sequences always had three keys repeated twice and one original key. The random sequences did not have such regularity; therefore results could have been influenced by this. However, over participants every key had the same amount of repetitions in the practiced sequences. Therefore, we do not think this influenced our results. 
Another point of consideration is that stimulus-response mappings varied in the two keyboard positions. For example, executing sequences with the left hand when the keyboard was on the left side of the body resulted in the little finger being closest to the body and the index finger being closest to the computer screen. However, executing sequences with the left hand when the keyboard was on the right side of the body resulted in the little finger being closest to the computer screen and the index finger being closest to body. It could be that the effects found in Experiment 1 were caused by this difference in stimulus-response mappings. Though, no effect of stimulus-response mappings were found in the random condition, therefore it is expected that the stimulus-response mappings were not responsible for the results of the first experiment. Experiment 2 was conducted to ascertain this.

\section{Experiment 2}

In this second experiment participants could not automatically react to stimuli because the whole sequence was indicated by one sequence-specific cue. In contrast to Experiment 1 sequences were initially learned verbally, which relies on a limited verbal working memory capacity. If performance in the test phase is independent of stimulusresponse mappings, Experiment 2 should replicate the results of Experiment 1.

Method

\section{Participants}

Thirty-two students (13 men, 19 women) from the University of Twente served as participants in this experiment. All were right-handed and between 18 and 26 years old. Participants received course credits for their participation.

\section{Apparatus and task}

The apparatus and task used in Experiment 2 were identical to Experiment 1, except that participants memorized two sequences of seven numbers at home before the experiment commenced. At the start of the experiment participants were tested on the memorization of the number-sequences by having them orally report the two sequences. All participants appeared to have correctly memorized the learned sequences. During the experiment the sequences were presented in the same way as in Experiment 1 except that participants reacted with the entire sequence to onset of just the first stimulus. This filled square corresponded with the first number of the learned sequence that had to be pressed and no further key-specific cues were given. For example, if the second square from the left was filled, the sequence that started with a ' 2 ' had to be pressed. At the end of a sequence feedback was given about which responses had been wrong (key press 1-7). If all responses had been correct no feedback was given. This time sequences were not distinguished by color of the first stimulus and there were no random sequences in the test phase.

\section{Procedure}

The procedure used in Experiment 2 was largely identical to the one in Experiment 1 except that during the first block of the practice phase participants had their sequences, written in numbers on a paper sheet, in front of them, to help them recall the sequences. After the first practice block the written sequences were removed and the participants were to complete the remaining three practice blocks without them. The instruction to the participants was to react as accurately and fast as possible to the filling of a square by subsequently pressing the appropriate series of keys of the sequence of digits they had learned at home. The mapping of the number to the finger presses was as follows; 1 referred to the left little finger, 2 to the left ring finger, 3 to the left middle finger, 4 to the left index finger, 5 referred to the right index finger, and so on.

\section{Data analysis}

The data analysis in Experiment 2 was identical to the data analysis in Experiment 1. The procedure of removing sequences in which the total RT lasted longer than the mean sequence execution time across participants and within blocks plus three standard deviations, removed $1.5 \%$ of the sequences. The data of block 1 of the practice phase of one participant was lost and therefore the calculated means of the first block is based on one participant less than the other block means. Mean RTs and arcsine transformed error rates were evaluated statistically by analysis of variance (ANOVA) with repeated measures, with in the practice phase Block (4) and Key (6) as within subject factors and in the test phase Hand (practiced left hand vs. unpracticed right hand), Position (practiced vs. unpracticed) and Key (6) as within subjects factors.

\section{Results}

\section{Practice phase}

Figure 4 shows that participants became faster with practice, $F(3,90)=165.3, P<0.001$, and that some keys were executed faster than others, $F(5,150)=12.3, P<0.001$. Participants made fewer errors in later blocks $[F(3,93)=8.3, P<0.001]$, while more errors were made 


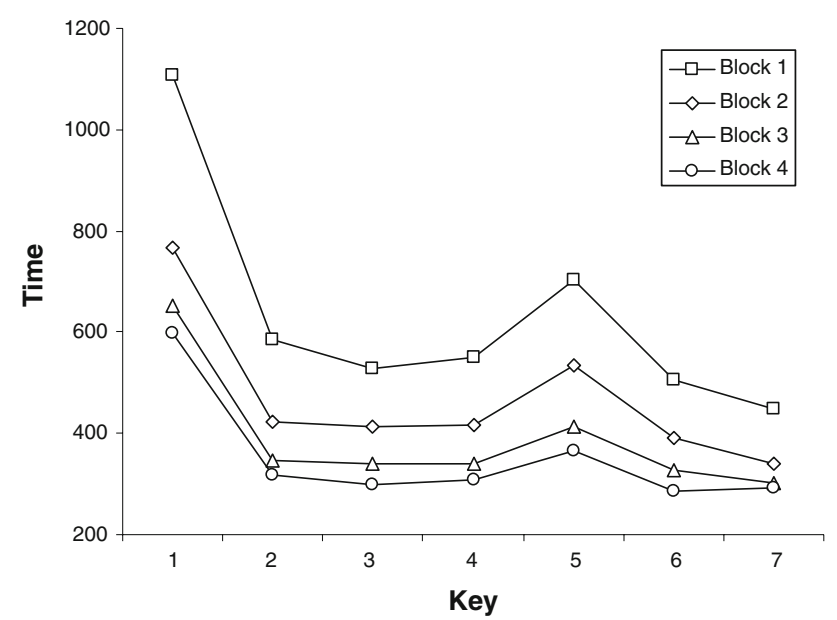

Fig. 4 Mean initiation time and mean RTs (in millisecond) in the four practice blocks of Experiment 2 as a function of key position

along the keys within the sequence $[F(5,155)=154.9$, $P<0.001]$. Furthermore, Fig. 4 shows that in the course of practice the sequences were less clearly segmented into two parts which was signified by the interaction between Block and Key, $F(15,450)=4.5, P<0.001$. There was also an interaction between Block and Key on errors, $F(15,465)=2.7, P<0.005$, which was difficult to interpret. Planned comparisons on RT showed that T5 was slower than T2, T3, T4, T6 and T7 separately for Blocks 1 through $4, F s(1,31)>6.3$, ps $<0.05$. This shows that in this experiment segmentation was already present in the first block of practice. This can be explained by limitations of verbal working memory with limited practice, which did not play a role in Experiment 1. Still, segmentation remained significant until the last block of practice and was of comparable size as in Block 4 of Experiment 1 (difference between Key 5 and the mean of the two adjacent keys in the last practice block was $54 \mathrm{~ms}$ in Experiment 1 and $66 \mathrm{~ms}$ in Experiment 2). Furthermore, in an additional ANOVA including Group as a independent variable there was again no significant interaction between Block, Key and Group $[F(15,435)=$ $1.0, P=0.44]$ confirming that the sequences were identically segmented over participants, despite the two groups executing two different sequences. Summarizing, participants learned the sequences, which were segmented in two parts.

\section{Test phase}

Participants were faster when executing sequences with the practiced hand than with the unpracticed hand, $F(1,31)=63.3, P<0.001$, and fewer errors were made with the practiced hand than with the unpracticed hand, $F(1,31)=14.0, P<0.001$ (6\% vs. $9 \%)$.
Figure 5 shows that some keys were executed faster than others, $F(5,155)=8.8, P<0.001$.In addition, later key presses had more errors, $F(5,155)=112.5, P<0.001$. Given the obvious segmentation of the sequences, RTs were analyzed with a 2 (Hand; practiced left hand vs. unpracticed right hand) $\times 2$ (Position; practiced vs. unpracticed position) $\times 2$ (Phase; T2, T3, T4, T6, $\mathrm{T} 7, \rightarrow$ execution, $\mathrm{T} 5 \rightarrow$ transition) $\times 2$ (Group; sequence vnbnvbc and nvcvncb vs. sequence bencbnv and cbvbcvn) repeated measure ANOVA. The transition phase was significantly slower than the execution phase, $F(1,31)=31.4$, $P<0.001$, and there was an interaction between Hand and Phase, $\quad F(1,30)=4.3, \quad p<0.05$. Planned comparisons showed that the practiced hand was faster than the unpracticed hand in both phases, $F s(1,31)>28.9$, $p s<0.001$. The difference in execution rate between the hands was $82 \mathrm{~ms}$ for the transition phase and $55 \mathrm{~ms}$ for the execution phase. Further planned comparisons were performed to investigate the relationship between the two phases and the keyboard position. Most importantly, for the practiced sequences executed with the practiced hand there was a significant difference between the practiced and the unpracticed hand position for the execution phase, $F(1,31)=16.1, P<0.001$, and not for the transition phase $F(1,31)=1.1, P>0.3$. Furthermore, the keyboard position did not influence the unpracticed hand in either phase, $F(1,31)>0.02, P>0.5$. See Fig. 5. Thus, only when using the practiced hand the position of the hand influenced the execution phase of the practiced sequences, but not the transition phase.

Taken together, the practice phase showed that the practiced sequences were learned and identically segmented

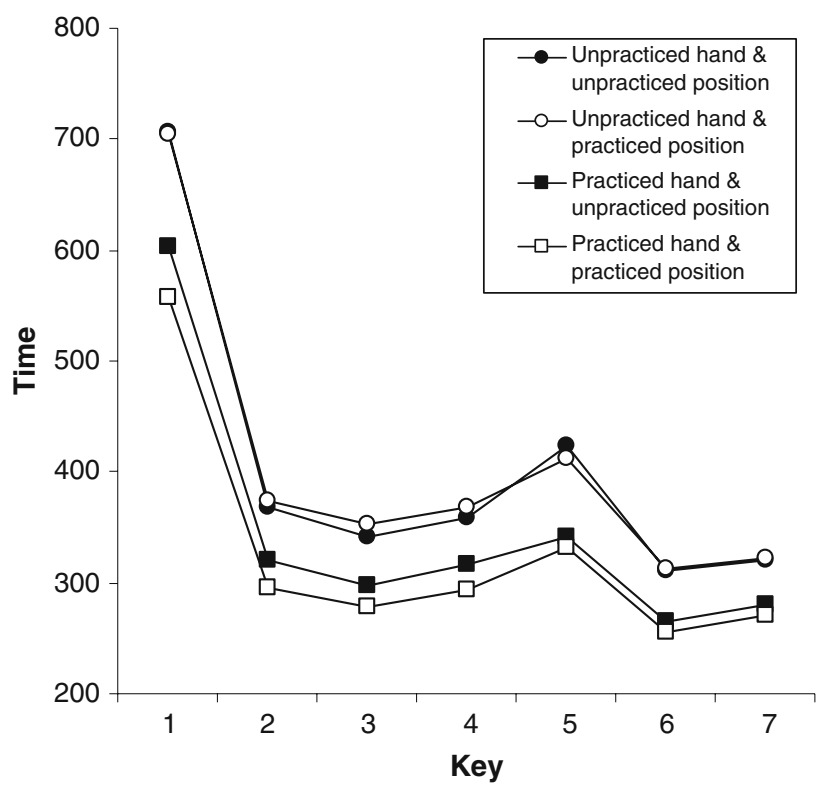

Fig. 5 Mean initiation time and mean RTs (in millisecond) in the test phase of Experiment 2 as a function of key position within the sequence, the hand used and the position of the hand used 
across participants and sequences. The test phase showed effector-dependent sequence learning and that the position of the practiced hand affected the execution of chunks during effector-dependent sequence learning of the practiced sequences, and not transition.

\section{Discussion}

The rationale for this second experiment was to replicate the results of the first experiment and to ascertain that the effects found in Experiment 1 had not been caused by different stimulus-response mappings in the two keyboard location conditions. The question remained; is sequence execution at more advanced levels of practice influenced by the hand used and by the position of the hand used? The initial way in which the sequences had been learned did not influence the eventual performance, thus refuting a stimulus-response mapping explanation for the results of Experiment 1 . However, RTs during the first practice block in Experiment 2 were about 150 ms larger compared with RTs during the first block in Experiment 1. This is probably caused by the need to retrieve each key press from verbal memory and translate it one by one.

While no effect of spatial position across keys on effector-dependent and effector-independent sequence learning was found, we do find an effect of spatial position on the execution phase of effector-dependent sequence learning. This indicates again that effector-dependent sequence knowledge includes both a location dependent (execution) and a location independent component (transition).

\section{General discussion}

In two experiments the influence of the position of the practiced and the unpracticed hand on DSP task performance was examined. In Experiment 1 participants learned the sequences by reacting to key-specific cues and in Experiment 2 participants learned the sequences by translating a numerical code. This difference left the eventual results unchanged, indicating that the effects found in Experiment 1 can not be explained by different stimulusresponse mappings in the two keyboard location conditions and that representations that develop during practice with the DSP task are independent of the initial way of learning.

In both experiments participants executed the practiced sequences faster with the practiced than with the unpracticed hand, indicating that participants developed effectordependent learning of the practiced sequences. This is in agreement with Hikosaka et al. (1999) who argued that at more advanced levels of learning sequences are executed increasingly effector-dependent. Furthermore, the models of Hikosaka et al. (1999) and Verwey (2003) suggest that effector-independent sequence learning is influenced by spatial coordinates because it is not related to specific body parts, while effector-dependent sequence learning is not influenced by spatial coordinates because it is related to specific body parts. However, in both experiments no effect of position across keys was found on effector-dependent or effector-independent sequence learning.

Still, the obvious segmentation of the sequences gave us the opportunity to investigate the influence of the position of the hand on the different phases of sequence execution. It appeared that chunk execution of effectordependent sequence learning was affected by the spatial position of the hand, while chunk transition was not. This suggests that slowing at T5 was indeed caused by other processes such as switching to a next chunk. So, the present experiments support the notion that at advanced skill levels sequence execution is based on several representations simultaneously, one being a representation that is both effector and position dependent and one being more general which is both effector and position independent. Furthermore, the present experiment suggests that chunk execution and chunk transition are represented by different codings, as only chunk execution was effected by the spatial position of the practiced hand. This agrees with the view that sequences are represented by different codings (Harrington et al. 2000; Hikosaka et al. 1999; Verwey 2003; Deroost et al. 2006).

Practice related shifts in representations are also mentioned in other studies. Hoffmann and Koch (1997) and Koch (2007) suggest that with practice sequence learning shift from a stimulus-based representation to a responsebased representation. This suggests that the representation that is effector and position independent is stimulus based, while the effector and position dependent representation is response based.

Finally, the present findings suggest that chunk execution of effector-dependent learning is in a body-centered (i.e., trunk, shoulder- or head-centered) reference frame, while chunk transition of effector-dependent learning and effector-independent learning were probably not in a bodycentered reference frame and perhaps in a world-based reference frame.

In conclusion, we argue that sequences can initially be learned either verbally or by responding to cues and that with additional practice an effector-dependent (perhaps motor) component develops in parallel to an effector-independent (perhaps spatial) component. We suggest that effector-dependent sequence learning consists of a location dependent component (chunk execution) and a location independent component (chunk transition). 
Acknowledgment The authors would like to thank Iring Koch and an anonymous reviewer for their comments on an earlier draft of this manuscript.

Open Access This article is distributed under the terms of the Creative Commons Attribution Noncommercial License which permits any noncommercial use, distribution, and reproduction in any medium, provided the original author(s) and source are credited.

\section{References}

Adam, J. J., Hommel, B., \& Umiltà, C. (2003). Preparing for perception and action (I): The role of grouping in the response-cuing paradigm. Cognitive Psychology, 46, 302-358.

Allport, D. A. (1980). Patterns and actions: Cognitive mechanisms are content-specific. In G. Claxton (Ed.), Cognitive psychology: New directions (pp. 26-64). London: Routledge and Kegan Paul.

Anderson, J. R. (1982). Acquisition of cognitive skill. Psychological Review, 89, 369-406.

Bapi, R. S., Doya, K., \& Harner, A. M. (2000). Evidence for effectorindependent and dependent representations and their differential time course of acquisition during motor sequence learning. Experimental Brain Research, 132, 149-162.

Bapi, R. S., Miyapuram, K. P., Graydon, F. X., \& Doya, K. (2006). fMRI investigation of cortical and subcortical networks in the learning of abstract and effector-specific representations of motor sequences. Neuroimage, 32, 714-727.

Colby, C. L., \& Goldberg, M. E. (1999). Space and attention in parietal cortex. Annual Review of Neuroscience, 22, 319-349.

Deroost, N., Zeeuws, I., \& Soetens, E. (2006). Effector-dependent and response location learning of probabilistic sequences in serial reaction time tasks. Experimental Brain Research, 171, 469-480.

Fitts, P. M. (1964). Perceptual-motor skill learning. In A. W. Melton (Ed.), Categories of human learning (pp. 243-285). New York: Academic.

Grafton, S. T., Hazeltine, E., \& Ivry, R. B. (1998). Motor sequence learning with the nondominant left hand. Experimental Brain Research, 146, 369-378.

Hardy, L., Mullen, R., \& Jones, G. (1996). Knowledge and conscious control of motor actions under stress. British Journal of Psychology, 87, 621-636.

Harrington, D. L., Rao, S. M., Haaland, K. Y., Bobholz, J. A., Mayer, A. R., Binderx, J. R., et al. (2000). Specialized neural systems underlying representations of sequential movements. Journal of Cognitive Neuroscience, 12, 56-77.

Heuer, H., \& Sangals, J. (1998). Task-dependent mixtures of coordinate systems in visuomotor transformations. Experimental Brain Research, 119, 224-236.

Hikosaka, O., Nakahara, H., Rand, M. K., Sakai, K., Lu, X., Nakamura, K., et al. (1999). Parallel neural networks for learning sequential procedures. Trends in Neuroscience, 22, 464-471.
Hoffmann, J., \& Koch, I. (1997). Stimulus-response compatibility and sequential learning in the serial reaction time task. Psychological Research, 60, 87-97.

Koch, I. (2007). Anticipatory response control in motor sequence learning: Evidence from stimulus-response compatibility. Human Movement Science, 26, 257-274.

Koch, I., \& Hoffmann, J. (2000a). The role of stimulus-based and response-based spatial information in sequence learning. Journal of Experimental Psychology: Learning, Memory and Cognition, 26, 863-882.

Koch, I., \& Hoffmann, J. (2000b). Patterns, chunks, and hierarchies in serial reaction-time tasks. Psychological Research, 63, 22-35.

Liu, T., Lungu, O. V., Waechter, T., Willingham, D. T., \& Ashe, J. (2007). Frames of reference during implicit and explicit learning. Experimental Brain Research, 180, 273-280.

Mayr, U. (1996). Spatial attention and implicit sequence learning: Evidence for independent learning of spatial and nonspatial sequences. Journal of Experimental Psychology: Learning, Memory and Cognition, 22, 350-364.

Park, J. H., \& Shea, C. H. (2005). Sequence learning: Response structure and effector transfer. The Quarterly Journal of Experimental Psychology, 58A, 387-419.

Rhodes, B. J., Bullock, D., Verwey, W. B., Averbaeck, B. B., \& Page, M. P. A. (2004). Learning and production of movement sequences: Behavioral, neurophysiological, and modeling perspectives. Human Movement Science, 23, 699-746.

Rieger, M. (2004). Automatic keypress activation in skilled typing. Journal of Experimental Psychology: Human Perception and Performance, 30, 555-565.

Schmidt, R. A. (1988). Motor control and learning: A behavioural emphasis. Champaign, IL: Human Kinetics.

Shaffer, L. H. (1991). Cognition and motor programming. In J. Requin \& G. E. Stelmach (Eds.), Turorials in motor neuroscience (pp. 371-383). Dordrecht: Kluwer.

Ungerleider, L. G., Doyon, J., \& Karni, A. (2002). Imaging brain plasticity during motor skill learning. Neurobiology of Learning and Memory, 78, 553-564.

Verwey, W. B. (2001). Concatenating familiar movement sequences: The versatile cognitive processor. Acta Psychologica, 106, 69-95.

Verwey, W. B. (2003). Processing modes and parallel processors in producing familiar keying sequences. Psychological Research, 67, 106-122.

Verwey, W. B., \& Clegg, B. A. (2005). Effector-dependent sequence learning in the serial RT task. Psychological Research, 69, 242-251.

Verwey, W. B., \& Eikelboom, T. (2003). Evidence for lasting sequence segmentation in the discrete sequence-production task. Journal of Motor Behavior, 35, 171-181.

Verwey, W. B., Lammens, R., \& Van Honk, J. (2002). On the role of the SMA in the discrete sequence production task: A TMS study. Neuropsychologia, 40, 1268-1276.

Verwey, W. B., \& Wright, D. L. (2004). Effector-independenst and effector-dependent learning in the discrete sequence production task. Psychological Research, 68, 64-70. 